\title{
New time-saving predictor algorithm for multiple breath washout in adolescents
}

\author{
Jonathan Grønbæk', Henrik Wegener Hallas', Lambang Arianto', Knud Pedersen², Arne Thomsen², Bo Lund Chawes' and \\ Hans Bisgaard'
}

BACKGROUND: Multiple breath washout (MBW) is an informative but time-consuming test. This study evaluates the uncertainty of a time-saving predictor algorithm in adolescents. METHODS: Adolescents were recruited from the Copenhagen Prospective Study on Asthma in Childhood (COPSAC $\left.{ }_{2000}\right)$ birth cohort. MBW trials were performed at 13 y of age with Innocor model Inn00400 using sulfur hexafluoride $\left(\mathrm{SF}_{6}\right)$ as tracer gas. Measurements were analyzed using a mixed model focusing on two prediction points doubling (t5\%) and quadrupling (t10\%) the standard end point (t2.5\%).

RESULTS: One hundred and seventy-two MBW trials conducted in 78 adolescents with and without asthma from COPSAC $_{2000}$ were included. At t10\%, the washout time (WoT) was reduced by $41 \%$, and an uncertainty of 0.159 lung clearance index ( $(\mathrm{Cl})$ units was introduced $( \pm 2 \mathrm{SD}), \pm 1.27)$. At $\mathrm{t} 5 \%$, the WoT was reduced by $25 \%$, with an uncertainty of $0.083 \mathrm{LCl}$ units $( \pm 0.558)$. The optimal prediction point, which led to most saved time and least uncertainty was $\mathrm{t} 5 \%$.

CONCLUSION: The predictor algorithm is capable of shortening the MBW test time but introduces an increasing uncertainty with earlier prediction points. This first-of-a-kind prediction algorithm holds promise in shortening the MBW test in children but should be used with caution in subjects with normal $\mathrm{LCl}$ values.

M ultiple breath washout (MBW) is a promising tool for measuring lung function $(1,2)$ requiring less cooperation, but longer measuring time than other methods, which highlights the need for time-saving procedures. The test investigates ventilation inhomogeneity of the lungs, which is currently measured as a lung clearance index (LCI). The MBW method used in this study measures LCI utilizing the nonresident tracer gas sulfur hexaflouride $\left(\mathrm{SF}_{6}\right)$ requiring a wash-in phase prior to the washout phase. One such MBW trial lasts $\sim 5$ minutes, dependent on the age, body size, and lung status of the subject. According to Consensus guidelines, three trials are needed to complete a test making MBW testing rather time consuming (3).
MBW has been shown to be more sensitive than spirometry in detecting structural lung disease accompanying, e.g., cystic fibrosis (1). While spirometry requires active patient collaboration, MBW is measured during tidal breathing and may therefore be more feasible for children $(4,5)$. However, some of the trials are typically interrupted due to impatience of the child. Additionally, changes in breathing pattern often occur at the end of the trial due to saliva production, dryness in the mouth, or lack of concentration, which can alter or even invalidate the test result. A reduction of test time therefore has the potential to increase success rate of the test by saving otherwise discarded trials, which could enable testing young children.

For historical reasons, the washout phase is proceeded until the end-tidal concentration of the tracer gas is $1 / 40$ of the starting concentration (6). In this study, we used MBW data from adolescents participating in the Copenhagen Prospective Study on Asthma in Childhood $\left(\right.$ COPSAC $_{2000}$ ) birth cohort to investigate the ability of a predictor algorithm to extrapolate LCI from higher end-tidal tracer gas concentrations. The aim was to evaluate the decrease in washout time (WoT) in relation to the introduced LCI uncertainty (LCI error (LCIe) at different points of prediction.

\section{RESULTS}

\section{Baseline}

Seventy-eight (46\% boys) of the 411 adolescents in the COPSAC $_{2000}$ cohort were included in the study at mean (SD) age 13 y $(0.64)$ and performed 216 trials. Fifty-four tests did not meet the predefined acceptability criteria and were excluded. Eighteen (21\%) subjects had asthma at the time of measurement. Mean (SD) forced expiratory volume in $1 \mathrm{~s} \mathrm{(FEV1)} \mathrm{/}$ forced vital capacity (FVC) was 0.88 (0.07), mean (SD) functional residual capacity (FRC) was 1.931 (0.45), and mean (SD) LCI was 6.22 units (0.51). Demographics, patient characteristics, and MBW baseline data are displayed in Table 1. Number of individuals and included trials at each prediction point are displayed in Table 2.

\footnotetext{
The first two authors contributed equally to this work.

'COPSAC, Copenhagen Prospective Studies on Asthma in Childhood, Herlev and Gentofte Hospital, University of Copenhagen, Copenhagen, Denmark; ${ }^{2}$ Innovision ApS,

Glamsbjerg, Denmark. Correspondence: Hans Bisgaard (bisgaard@copsac.com)

Received 30 March 2015; accepted 19 January 2016; advance online publication 20 April 2016. doi:10.1038/pr.2016.57
} 


\section{Articles | Gronbreket al.}

\section{Error and Saved Time}

In Figure 1, the LCIe are shown with prediction limits and SDs. The lowest mean LCIe is found at $t 4 \%$ and the highest LCIe is found at $\mathrm{t} 10 \%$. At all prediction points, the mean error is positive, and the SD is increasing from $\mathrm{t} 4 \%$ to $\mathrm{t} 10 \%$.

In Table 3, the decrease in WoT is shown at the different prediction points as saved time (ST). The lowest ST is found at $\mathrm{t} 4 \%$ and the highest ST is found at $\mathrm{t} 10 \%$. In Table $1, \mathrm{MBW}$

Table 1. Baseline

\begin{tabular}{|c|c|}
\hline No. of subjects & $n=78$ \\
\hline \multicolumn{2}{|l|}{ Antropometrics } \\
\hline \multirow[t]{2}{*}{ Gender (\%male) } & 46 \\
\hline & Mean (SD) \\
\hline Age (years) & $13(0.64)$ \\
\hline Height (cm) & $161.8(8.4)$ \\
\hline Weight (kg) & $52.0(10.6)$ \\
\hline \multicolumn{2}{|l|}{ Spirometry } \\
\hline $\mathrm{FEV}_{1} / \mathrm{FVC}$ & $0.88(0.07)$ \\
\hline \multicolumn{2}{|l|}{ MBW } \\
\hline FRC (I.) & $1.93(0.45)$ \\
\hline $\mathrm{LCl}$ & $6.22(0.51)$ \\
\hline \multirow[t]{2}{*}{ WoT (s) } & $93.2(22.9)$ \\
\hline & Median (IQR) \\
\hline $\mathrm{CV}_{\mathrm{LCI}}(\%)$ & $3.2(2.0 ; 4.5)$ \\
\hline $\mathrm{CV}_{\text {NewLCI5 }}(\%)$ & $4.7(2.1 ; 6.3)$ \\
\hline $\mathrm{CV}_{\text {NewLC110 }}(\%)$ & $5.3(2.3 ; 9.5)$ \\
\hline $\mathrm{CV}_{\mathrm{FRC}}(\%)$ & $2.7(1.7 ; 4.4)$ \\
\hline
\end{tabular}

Demographics, patient characteristics, and MBW baseline data.

$\mathrm{CV}$,coefficient of variation; FEV1, forced expiratory volume in $1 \mathrm{~s} ; \mathrm{FVC}$, forced vital

capacity; FRC, funtional residual capacity; IQR, interquartile range; LCI, lung clearance index; MBW, multiple breath washout; WoT, washout time. data are shown with the coefficient of variation $(\mathrm{CV})$ of FRC, original LCI $\left(\mathrm{LCI}_{\mathrm{Org}}\right)$, LCI at $\mathrm{t} 5 \%\left(\mathrm{LCI}_{\mathrm{New} 5}\right)$, and LCI at $\mathrm{t} 10 \%$ $\left(\mathrm{LCI}_{\mathrm{New10}}\right)$. Median (interquartile range (IQR)) $\mathrm{CV}$ of $\mathrm{LCI}_{\mathrm{Org}}$ is $3.2 \%(2.0 ; 4.5)$, where median (IQR) CV for prediction point $\mathrm{t} 5 \%$ and $\mathrm{t} 10 \%$ is $4.7 \%(2.1 ; 6.3)$ and $5.3 \%(2.3 ; 9.5)$, respectively.

\section{Prediction}

The ST/highest prediction limit (HPL) ratio is highest at $\mathrm{t} 5 \%$ indicating that this is the optimal extrapolation point (Table 3).

In Figure 2, the difference between $\mathrm{LCI}_{\mathrm{New}}$ and $\mathrm{LCI}_{\mathrm{Org}}$ is plotted against $\mathrm{LCI}_{\mathrm{Org}}$ to examine for systematic errors in the algorithm. At $\mathrm{t} 10 \%$, the differences are distributed over a larger range than at $\mathrm{t} 5 \%$. At $\mathrm{t} 5 \%$, the distribution is not associated with the magnitude of the mean $\mathrm{LCI}_{\mathrm{Org}}$, whereas at $\mathrm{t} 10 \%$, the distribution seems more scattered in the higher values of LCI (7 to 8), with fewer observations near zero. However, logarithmic transformation of the differences did not improve the distribution (data not shown).

When extrapolating from $t 10 \%$, we found a mean LCIe $( \pm 2$ SD) of $0.159( \pm 1.27)$ and a mean ST of $38.6 \mathrm{~s}$. In comparison, when extrapolating from $\mathrm{t} 5 \%$, we found a mean LCIe of 0.083

Table 2. Number of tests

\begin{tabular}{lcc}
\hline Time point & Individuals & Total trials \\
\hline t10\% & 77 & 162 \\
t9\% & 77 & 156 \\
t8\% & 77 & 153 \\
t7\% & 78 & 154 \\
t6\% & 76 & 149 \\
t5\% & 74 & 144 \\
t4\% & 67 & 134 \\
\hline
\end{tabular}

Amount of tests included at different time points.

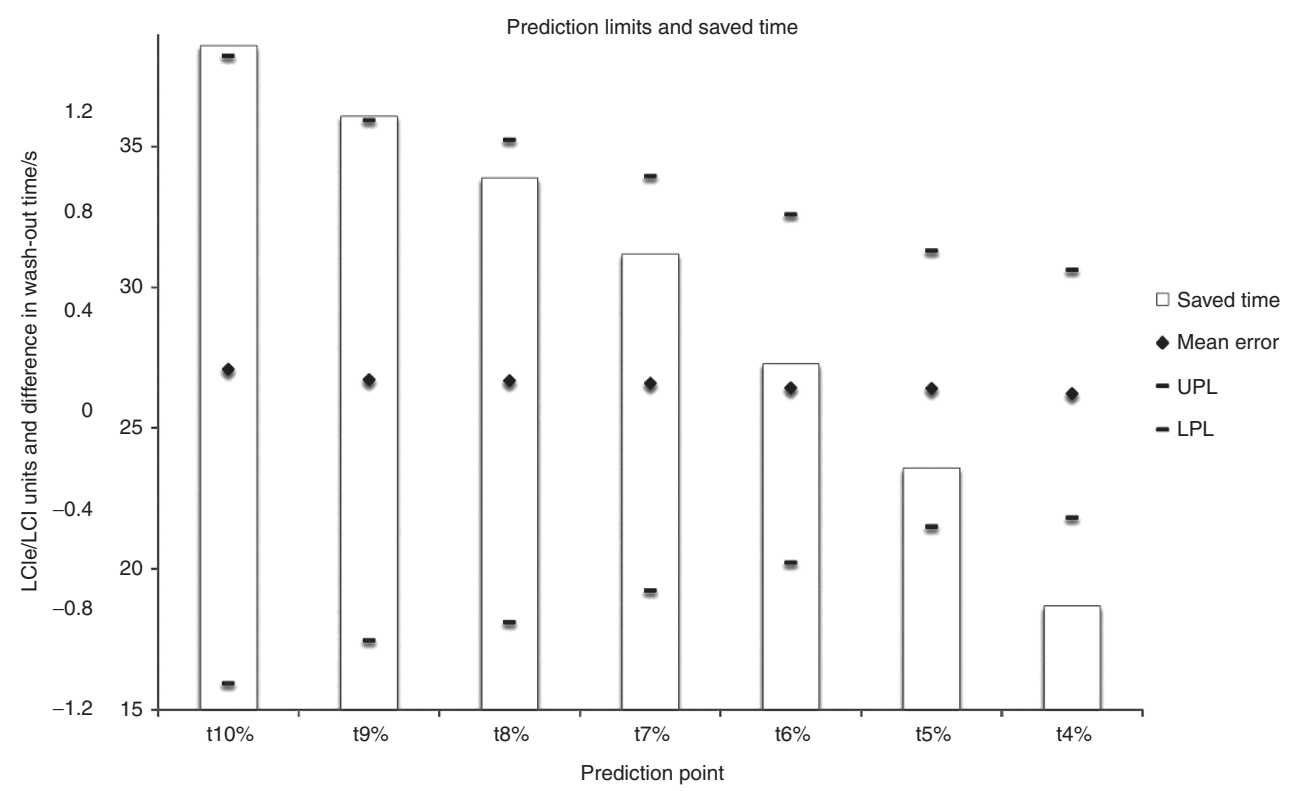

Figure 1. Mean prediction error (new - original) with $95 \%$ prediction limits in LCl units (left axis) at different prediction points. The column is decrease in washout time in seconds (right axis). LCl, lung clearance index; LPL,lower prediction limit; UPL, upper prediction limit. 
$( \pm 0.558)$ and a mean ST of $23.6 \mathrm{~s}$. At $\mathrm{t} 5 \%$ where ST/HPL is highest, the LCIe $( \pm 2 \mathrm{SD})$ equals $1.3 \%( \pm 9 \%)$ of the mean LCI and the WoT is reduced with $25.3 \%$.

\section{DISCUSSION}

The proposed predictor algorithm is capable of shortening the MBW test procedure and provides an estimate of the LCI with a mean LCIe $( \pm 2$ SD) of $1.3 \%( \pm 9 \%)$ and a reduction in mean WoT of $25.3 \%$ at the optimal prediction point.

At all prediction points the mean LCIe is positive, and the $\mathrm{SD}$ is increasing from $\mathrm{t} 4 \%$ to $\mathrm{t} 10 \%$. This implies that the algorithm is overestimating LCI and indicates that earlier prediction points increase the uncertainty. To determine the optimal prediction point to use for extrapolation, it is relevant to evaluate the amount of saved time in relation to the LCIe and the $\mathrm{SD}$ of this. HPL gives an idea of the most extreme error the algorithm introduces, and from our data, a direct correlation

Table 3. Prediction

\begin{tabular}{|c|c|c|c|}
\hline $\begin{array}{l}\text { Stop } \\
\text { point }\end{array}$ & $\begin{array}{c}\text { LCle, mean } \\
\text { (SD) (LCl units) }\end{array}$ & $\begin{array}{l}\text { ST, mean } \\
(\mathrm{SEM})(\mathrm{s})\end{array}$ & $\mathrm{ST} / \mathrm{HPL}$ \\
\hline $\mathrm{t} 4 \%$ & $0.062(0.250)$ & $18.7(0.85)$ & 33.6 \\
\hline $\mathrm{t} 5 \%$ & $0.083(0.279)$ & $23.6(1.13)$ & 37.2 \\
\hline t6\% & $0.085(0.352)$ & $27.3(1.28)$ & 35.0 \\
\hline $\mathrm{t} 7 \%$ & $0.105(0.419)$ & $31.3(1.44)$ & 33.6 \\
\hline $\mathrm{t} 8 \%$ & $0.115(0.488)$ & $33.9(1.56)$ & 31.5 \\
\hline t9\% & $0.228(0.526)$ & 36.1 (1.69) & 31.2 \\
\hline $\mathrm{t} 10 \%$ & $0.159(0.635)$ & $38.6(1.82)$ & 27.3 \\
\hline
\end{tabular}

HPL, highest prediction limit; LCI, lung clearance index; LCle, LCl error; ST, saved time.

a

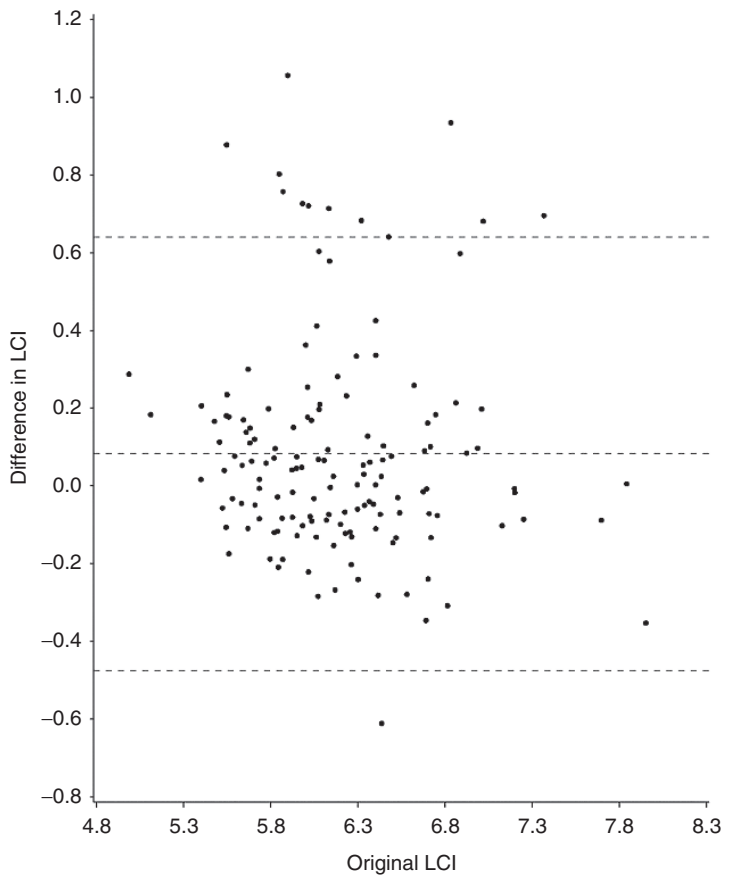

of the saved time and HPL suggests that the optimal point of prediction is $t 5 \%$. At this prediction point, the HPL is $0.64 \mathrm{LCI}$ units, which is considered a high inaccuracy in subjects with normal LCI values.

The median $\mathrm{CV}$ for $\mathrm{LCI}_{\mathrm{Org}}$ in our study was $3.2 \%$, which is comparable to other studies reporting $\mathrm{CV}$ for $\mathrm{LCI}_{\mathrm{Org}}$ from 4 to $9 \%(7-10)$. This implies that the MBW measurement itself is affected by some uncertainty, which should be taken into account when evaluating the algorithm. The CV is higher for extrapolated LCI values compared to the $\mathrm{CV}$ of $\mathrm{LCI}_{\mathrm{Org}}$, underlining the uncertainty introduced by the algorithm.

Lung function measurements are commonly used in a clinical setting to distinguish between normal and abnormal subjects and to monitor disease progressing. Our study was cross-sectional, why we cannot evaluate how the algorithm performs over time in the same subject.

Shortening of the MBW test time has been the topic of interest of other studies. Yammine et al. (11) examined sensitivity and specificity in children with cystic fibrosis when stopping the washout at $5 \%$ of the starting concentration. They found a similar or improved sensitivity and specificity with this prediction point compared with the traditional end point of $2.5 \%$ and proposed this as a way of shortening test time. The average of saved measuring time in cystic fibrosis patients was 30 vs. $21 \%$ in controls (11), which is comparable to our findings of a $25 \%$ reduction of WoT in a mixed cohort of asthmatics and healthy adolescents.

The predictor algorithm is capable of shortening the washout phase in each trial by $20-30 \mathrm{~s}$ when extrapolating from $\mathrm{t} 5 \%$. This may be considered negligible but actually accounts for at least a $25 \%$ reduction of the washout phase and $10-15 \%$

b

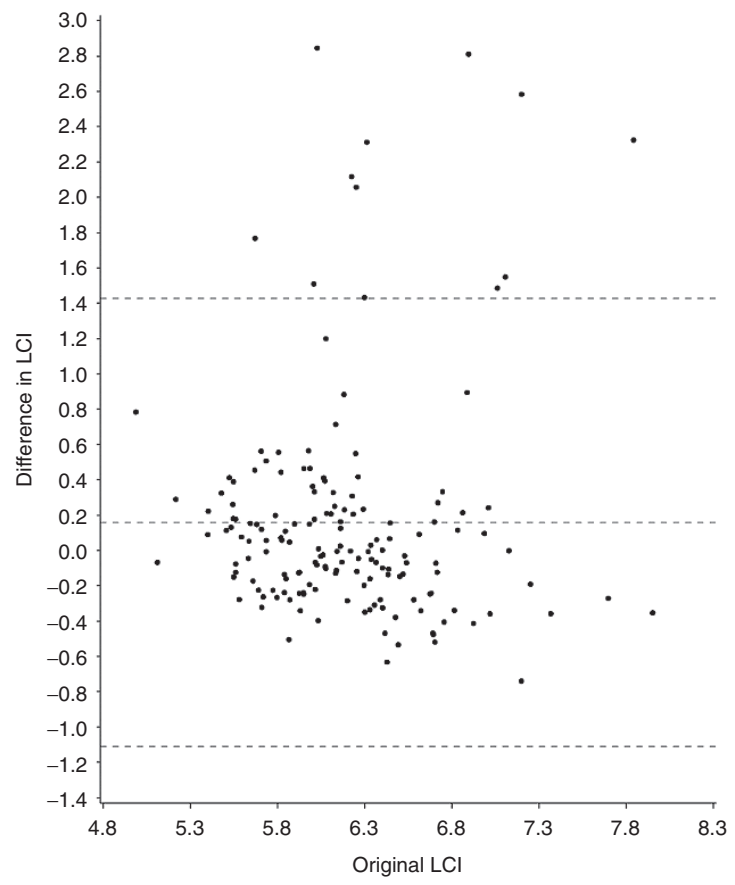

Figure 2. Bland-Altman plot for prediction points (a) $\mathrm{t} 5 \%$ and (b) $\mathrm{t} 10 \%$, doubling and quadrupling the standard prediction point of $\mathrm{t} 2.5 \%$, i.e., $1 / 40$ of the starting concentration. The difference between predicted (new) and original (org) data is plotted against original result. 


\section{Articles $\mid$ Gronberketal.}

reduction of the entire test time, which may facilitate and ease testing of young children. Moreover, the wash-in phase will also be shortened, as the concentration of tracer gas at the beginning of the wash-in will be higher.

The algorithm, which is based on washout of $\mathrm{SF}_{6}$ tracer gas, approved for use with Innocor (Innovision ApS, Glamsbjerg, Denmark) in the European Union and the United States, could be modified to include washout of other tracer gasses such as $\mathrm{N}_{2}$.

In conclusion, this study investigates a new approach to the MBW method using a novel predictor algorithm with the potential to save test time and avoid discarded tests in impatient children. The algorithm is capable of shortening the test with at least $10-15 \%$ with a mean LCIe ( \pm 2 SD) of $1.3 \%( \pm 9 \%)$ but introduces a potential uncertainty of around 0.6 LCI units.

\section{METHODS}

\section{Study Population}

Participants in this study were recruited from the COPSAC ${ }_{2000}$ cohort (12), which is a prospective, longitudinal, birth-cohort study of 411 infants born to asthmatic mothers in 1998-2001. The children were enrolled at 1 mo of age excluding children with severe congenital abnormality, gestational age $<36 \mathrm{wk}$, and any lung symptoms prior to enrolment. The children were followed closely from birth with scheduled clinic visits every half-year until age $7 \mathrm{y}$ and again at age $13 \mathrm{y}$. All the MBW measurements utilized in this study were conducted routinely during the scheduled $13-y$ visit at the COPSAC clinic. The 78 included participants were the ones who had completed MBW measurements by the time of data analysis.

Lung symptoms were recorded in daily diaries fulfilled from birth by the parents. Asthma was defined according to a strict predefined algorithm based on these lung symptom recordings and response to treatment and was exclusively diagnosed by the pediatricians working at the COPSAC clinic. Adolescents without asthma were defined as healthy in this study.

\section{MBW Measurements}

The Innocor model Inn00400, (Innovision ApS), which was used to perform MBW assessments in this study, was validated as described by Gonem et al. (13). It was calibrated on a daily basis according to manufacturer's guidelines and used an almost fully automatic software that ensured an equal measuring technique independent of the observer. The device has a re-breathing valve unit with connections to a re-breathing bag for the wash-in phase of the tracer gas $\mathrm{SF}_{6}$, a flow meter connected to a mouthpiece via a bacterial filter, and room air. MiniValve with a pre-capillary dead space of $62.3 \mathrm{ml}$ was inserted in the re-breathing valve unit. The mean (SD) weight of the participants was $52.0 \mathrm{~kg}$ (10.6) giving a mean pre-capillary dead space per $\mathrm{kg}$ body weight (SD) of $1.25 \mathrm{ml} / \mathrm{kg}(0.24)$.

For the wash-in phase, the device supplied a gas mixture containing $0.2 \% \mathrm{SF}_{6}$ and $35.5 \%$ oxygen $\left(\mathrm{O}_{2}\right)$ among others and used a scrubber to clear the produced carbon dioxide $\left(\mathrm{CO}_{2}\right)$. Participants wore noseclips and breathed through mouthpieces (small, $22 \mathrm{~mm}$, Hans Rudolph, Shawnee, KS) attached to a flow meter (4719 series Hans Rudolph) until gas equilibrium was reached, i.e., the variation of the $\mathrm{SF}_{6}$ concentration between inspiration and expiration was below $2 \%$. The connections switched from re-breathing bag to room air at the first exhalation of the washout phase. The participants breathed room air until the end tidal concentration of $\mathrm{SF}_{6}$ had reached a level below $1 / 40$ th of the starting concentration in three consecutive breaths. Only successfully finished trials were included. The outcomes that were used in the analysis were LCI, FRC, and WoT.

The collected data were sent to Innovision ApS, where the algorithm was used to extrapolate LCI values. Thereafter, raw data and the extrapolated values were sent to the COPSAC clinic, where the statistical analysis was performed, and the results were interpreted. Analysis was focused on two prediction points doubling ( $\mathrm{t} 5 \%)$ and quadrupling (t10\%) the standard finish point of the trial $(2.5 \%$ of the initial tracer gas concentration).

\section{Predictor Algorithm}

The algorithm was designed to predict the LCI and FRC at 2.5\% $(1 / 40)$ of the starting concentration at prediction points between 10 and $4 \%$ of the starting concentration. The washout part of $\mathrm{SF}_{6}$ was first normalized to an even breathing tidal volume using the phase three slope of the $\mathrm{SF}_{6}$. Then, a best-fit-curve through the last nine endtidal values was extrapolated to the normal $2.5 \%$, and the estimated expired cumulated volume (VCE) was found. A similar best-fit-curve was done on the $\mathrm{SF}_{6}$ volume of each breath and by extrapolating to the estimated VCE the total volume of expired $\mathrm{SF}_{6}$ was estimated, and the FRC was determined. Finally, the predicted LCI was calculated as the estimated VCE divided by the estimated FRC.

\section{Statistics}

A predefined acceptability criterion was made for MBW trials in the same subject: $\left(\mathrm{FRC}_{\mathrm{x}}-\mathrm{FRC}_{\text {mean }}\right) / \mathrm{FRC}_{\text {mean }}<4.8 \%$, which is in agreement with the most narrow acceptability criterion of ERS/ATS consensus statement (3). If all trials from an individual varied $>4.8 \%$, the individual was excluded.

LCIe is defined as the difference between $\mathrm{LCI}_{\mathrm{New}}$ and $\mathrm{LCI}_{\mathrm{Org}}\left(\mathrm{LCI}_{\mathrm{New}}\right.$ $\left.-\mathrm{LCI}_{\mathrm{Org}}\right)$. ST is defined by the difference between the original WoT and the time at prediction. The HPL is the numerical value of LCIe plus $2 \mathrm{SD}$. The ratio ST/HPL is calculated by dividing saved time with HPL. It is used to find the optimal prediction point regarding the amount of saved time in relation to the size of the error obtained when using the algorithm.

Individual $\mathrm{CV}$ was calculated by the $\mathrm{SD}$ of all trials of the individual divided by the mean. A median $\mathrm{CV}$ and IQR were calculated for LCI $\mathrm{FRC}, \mathrm{LCI}_{\mathrm{New5}}$, and $\mathrm{LCI}_{\mathrm{New10}}$, as the values were not normally distributed.

Measurements of LCIe and ST were analyzed using a mixed model with the individual as a random effect in order to take account of the repeated measurements on the same individual. Mean values for LCIe and ST were calculated as the overall estimate of the intercept in this model. These were compared to the mean LCI and mean WoT to calculate the percentage deviation and reduction. For the LCIe, SDs of the individual observations were calculated by summing up between- and within-individual variance using mixed model estimates. Prediction limits were defined as the $95 \%$ confidence limits on the individual observations calculated using the SD for individual observations. For saved time, SE of the mean saved time is provided by estimating the SE of the intercept in the mixed model.

Prediction at $\mathrm{t} 5 \%$ and $\mathrm{t} 10 \%$ were chosen for further analyses as the concentrations are two and four times the concentration at the end of a conventional test (1/40 of the starting concentration), respectively.

Prediction point-dependent LCIe was assessed using plots showing mean and upper and lower prediction limits. Prediction pointdependent saved time was compared to LCle. Agreement between $\mathrm{LCI}_{\mathrm{Org}}$ and $\mathrm{LCI}_{\mathrm{New}}$ thus the LCle, was assessed using Bland-Altman plots in $\mathrm{t} 5 \%$ and $\mathrm{t} 10 \%$ with limits of agreement (mean $\pm 2 \mathrm{SD}$ ) shown as dotted lines (14).

All statistics on algorithm data were carried out using SAS version 9.3 and SAS Enterprise Guide 5.1 version 5.100.0.12019 (SAS, Cary, $\mathrm{NC})$ at the COPSAC clinic.

\section{Ethics}

The Copenhagen Ethics Committee (KF 01-289/96) and The Danish Data Protection Agency (2008-41-2434) approved the study, and informed consent was obtained from both parents at enrollment.

\section{STATEMENT OF FINANCIAL SUPPORT}

COPSAC is funded by private and public research funds all listed on www. copsac.com. The Lundbeck Foundation, Copenhagen, Denmark; Danish State Budget, Copenhagen, Denmark; Danish Council for Strategic Research, Copenhagen, Denmark; The Danish Council for Independent Research, Copenhagen, Denmark; and The Capital Region Research Foundation, Hillerød, Denmark, have provided core support for COPSAC. The funding agencies did not have any influence on study design, data collection, data analysis, decision to publish, or preparation of the manuscript. 
Disclosure: The authors declare no conflict of interests.

\section{REFERENCES}

1. Gustafsson PM, De Jong PA, Tiddens HA, Lindblad A. Multiple-breath inert gas washout and spirometry versus structural lung disease in cystic fibrosis. Thorax 2008;63:129-34.

2. Macleod KA, Horsley AR, Bell NJ, Greening AP, Innes JA, Cunningham S. Ventilation heterogeneity in children with well controlled asthma with normal spirometry indicates residual airways disease. Thorax 2009;64: 33-7.

3. Robinson PD, Latzin $\mathrm{P}$, Verbanck $\mathrm{S}$, et al. Consensus statement for inert gas washout measurement using multiple- and single- breath tests. Eur Respir J 2013;41:507-22.

4. Gustafsson PM. Inert gas washout in preschool children. Paediatr Respir Rev 2005;6:239-45.

5. Beydon N, Davis SD, Lombardi E, et al.; American Thoracic Society/European Respiratory Society Working Group on Infant and Young Children Pulmonary Function Testing. An official American Thoracic Society/ European Respiratory Society statement: pulmonary function testing in preschool children. Am J Respir Crit Care Med 2007;175:1304-45.

6. Robinson PD, Goldman MD, Gustafsson PM. Inert gas washout: theoretical background and clinical utility in respiratory disease. Respiration 2009;78:339-55.
7. Zwitserloot A, Fuchs SI, Müller C, Bisdorf K, Gappa M. Clinical application of inert gas multiple breath washout in children and adolescents with asthma. Respir Med 2014;108:1254-9.

8. Amin R, Subbarao P, Jabar A, et al. Hypertonic saline improves the LCI in paediatric patients with CF with normal lung function. Thorax 2010;65:379-83.

9. Fuchs SI, Eder J, Ellemunter H, Gappa M. Lung clearance index: normal values, repeatability, and reproducibility in healthy children and adolescents. Pediatr Pulmonol 2009;44:1180-5.

10. Aurora P, Gustafsson P, Bush A, et al. Multiple breath inert gas washout as a measure of ventilation distribution in children with cystic fibrosis. Thorax 2004;59:1068-73.

11. Yammine S, Singer F, Abbas C, Roos M, Latzin P. Multiple-breath washout measurements can be significantly shortened in children. Thorax 2013;68:586-7.

12. Bisgaard H. The Copenhagen Prospective Study on Asthma in Childhood (COPSAC): design, rationale, and baseline data from a longitudinal birth cohort study. Ann Allergy Asthma Immunol 2004;93:381-9.

13. Gonem S, Singer F, Corkill S, Singapuri A, Siddiqui S, Gustafsson P. Validation of a photoacoustic gas analyser for the measurement of functional residual capacity using multiple-breath inert gas washout. Respiration 2014;87:462-8.

14. Bland JM, Altman DG. Statistical methods for assessing agreement between two methods of clinical measurement. Lancet 1986;1:307-10. 